\title{
Brilliant GeV electron beam with narrow energy spread generated by a laser plasma accelerator
}

\author{
Ronghao Hu, ${ }^{1}$ Haiyang Lu, ${ }^{1,3, *}$ Yinren Shou, ${ }^{1}$ Chen Lin, ${ }^{1}$ Hongbin Zhuo, ${ }^{2}$ \\ Chia-erh Chen, ${ }^{1}$ and Xueqing Yan ${ }^{1,3, \dagger}$ \\ ${ }^{1}$ State Key Laboratory of Nuclear Physics and Technology, and Key Laboratory of HEDP of the Ministry \\ of Education, CAPT, Peking University, Beijing 100871, China \\ ${ }^{2}$ National University of Defense Technology, Changsha 410073, China \\ ${ }^{3}$ Collaborative Innovation Center of Extreme Optics, Shanxi University, Taiyuan, Shanxi 030006, China
}

(Received 2 February 2016; published 6 September 2016)

\begin{abstract}
The production of $\mathrm{GeV}$ electron beam with narrow energy spread and high brightness is investigated using particle-in-cell simulations. A controlled electron injection scheme and a method for phase-space manipulation in a laser plasma accelerator are found to be essential. The injection is triggered by the evolution of two copropagating laser pulses near a sharp vacuum-plasma transition. The collection volume is well confined and the injected bunch is isolated in phase space. By tuning the parameters of the laser pulses, the parameters of the injected electron bunch, such as the bunch length, energy spread, emittance and charge, can be adjusted. Manipulating the phase-space rotation with the rephasing technique, the injected electron bunch can be accelerated to $\mathrm{GeV}$ level while keeping relative energy spread below $0.5 \%$ and transverse emittance below $1.0 \mu \mathrm{m}$. The results present a very promising way to drive coherent $\mathrm{x}$-ray sources.
\end{abstract}

DOI: 10.1103/PhysRevAccelBeams.19.091301

\section{INTRODUCTION}

Laser plasma accelerators (LPAs) are of great interest because of their abilities to sustain extremely large acceleration fields, typically 1000-fold higher than that of the conventional accelerators [1-4]. One promising application of LPAs is driving compact $\mathrm{x}$-ray free electron lasers (FELs) [5-7]. Modern FELs based on conventional accelerators are the brightest $\mathrm{x}$-ray sources ever and are indispensable tools for material science, medicine, biology and chemistry. Their applications are limited by the sizes and expenses of the driving accelerators, whereas LPAs may overcome. One of the biggest obstacles towards LPAFEL is the large energy spread of the electron beams, which is required to be as low as the Pierce parameter in FELs $[5,6]$. In the laser wakefield acceleration (LWFA) regime, self-injected electron beams with energy up to $4.2 \mathrm{GeV}$ have been experimentally demonstrated [8-11], but the relative energy spread is typically a few percent, which is more than 10 times larger than the Pierce parameter. The self-injection mechanism is the most basic injection method, and has been investigated thoroughly in theories [12-18], simulations [19-23] and experiments [24-32]. The injection length of self-injection is typically a few hundreds of microns [15,23], and the divergence of injected

\footnotetext{
*hylu@pku.edu.cn

†x.yan@pku.edu.cn
}

Published by the American Physical Society under the terms of the Creative Commons Attribution 3.0 License. Further distribution of this work must maintain attribution to the author $(s)$ and the published article's title, journal citation, and DOI. electrons is typically a few milliradians [33-35]. Several controlled injection schemes were proposed, such as the density transition injection [36-39], multipulse colliding injection [40-44] and ionization injection [45-54], but the production of $\mathrm{GeV}$ electron beam with narrow energy spread $(\lesssim 0.5 \%)$ remains to be challenging.

In this paper, we show that with a controlled injection scheme and a method for phase-space manipulation, brilliant $\mathrm{GeV}$ electron beam with narrow energy spread can be generated by a LPA. In the following discussion, we focus on the injection and acceleration in the first plasma bubble right after the driving laser pulse, because the first bubble is more stable and scalable [12-23]. Controlling the electron injection process is the key to improve beam qualities. Among all the controlled injection methods, we choose the dual-pulse copropagating injection (DPCI) for the outstanding beam qualities and tunabilities [55]. DPCI utilizes two colinear laser pulses with proper time delay and a relatively sharp vacuum-plasma transition (VPT). The front pulse, or the driver pulse, with the amplitude under the threshold for self-injection and a spot size close to the matched condition for self-guiding [16-18], is used to pump the wakefields for long distance acceleration without dark currents [21,23]. The trailing pulse with shorter duration, or the injector pulse, is tightly focused to modulate the wakefields to induce localized injection near the VPT. As one can see, the roles of the two copropagating pulses are different from those of Ref. [55] and thus the injection mechanism is fundamentally different. With the rephasing technique [56-59], the phase-space dynamics of 
injected electrons can be manipulated and narrow energy spread electron beam at $\mathrm{GeV}$ level can be obtained.

To investigate the injection and acceleration process, we carried out two-dimensional (2D) and three-dimensional (3D) particle-in-cell (PIC) simulations using the KLAP [60] and EPOCH [61] codes. The total spatiotemporal profile of two linearly polarized femtosecond Gaussian pulses propagating in vacuum along the $x$ axis can be written as

$$
\begin{aligned}
\boldsymbol{a} & =a_{0} \exp \left\{-\frac{\left[t-t_{\text {peak } 0}-r^{2} /\left(2 c R_{0}\right)-x / c\right]^{2}}{2 \tau_{0}^{2}}\right\} \cdot \exp \left(-\frac{r^{2}}{\sigma_{0}^{2}}\right) \cdot \\
& \sin \left[\omega_{0} \cdot\left(t-\frac{r^{2}}{2 c R_{0}}-\frac{x}{c}\right)+\phi_{G 0}+\phi_{0}\right] \boldsymbol{u}_{0} \\
& +a_{1} \exp \left\{-\frac{\left[t-t_{\text {peak } 1}-r^{2} /\left(2 c R_{1}\right)-x / c\right]^{2}}{2 \tau_{1}^{2}}\right\} \cdot \exp \left(-\frac{r^{2}}{\sigma_{1}^{2}}\right) . \\
& \sin \left[\omega_{1} \cdot\left(t-\frac{r^{2}}{2 c R_{1}}-\frac{x}{c}\right)+\phi_{G 1}+\phi_{1}\right] \boldsymbol{u}_{\mathbf{1}} .
\end{aligned}
$$

The subscripts 0 and 1 denote the driver pulse and the injector pulse respectively. $\boldsymbol{a}=e \boldsymbol{E}_{\perp} /\left(m_{e} \omega c\right) \approx 0.311$. $\lambda[\mu \mathrm{m}] \cdot \boldsymbol{E}_{\perp}[\mathrm{TV} / \mathrm{m}]$ is the normalized transverse electric field, $e$ is the elementary charge, $\boldsymbol{E}_{\perp}$ is the laser transverse electric field, $m_{e}$ is the electron rest mass, $\omega=2 \pi c / \lambda$ is the carrier angular frequency, $\lambda$ is the carrier wavelength, $c$ is the light velocity in vacuum, and $\boldsymbol{u}$ is the unit vector of the polarization direction. For two-dimensional simulations, $a=a_{\text {peak }} \sqrt{\sigma_{w} / \sigma}, r=\sqrt{y^{2}}, \phi_{G}=\arctan \left[\left(x-x_{f}\right) / x_{r}\right] / 2$ and for three-dimensional simulations $a=a_{\text {peak }} \sigma_{w} / \sigma$, $r=\sqrt{y^{2}+z^{2}}, \quad \phi_{G}=\arctan \left[\left(x-x_{f}\right) / x_{r}\right], \quad$ where $a$ describes the evolution of laser amplitude due to the focusing and defocusing, $\phi_{G}$ is the Gouy phase,

$a_{\text {peak }}$ is the peak amplitude at the focus, $\sigma=$ $\sigma_{w} \sqrt{1+\left[\left(x-x_{f}\right) / x_{r}\right]^{2}}$ is the spot size at $x, \sigma_{w}$ is the waist size $\left(1 / \mathrm{e}^{2}\right.$ focal radius measured by intensities, where $\mathrm{e}$ is the natural constant), $x_{f}$ is the longitudinal coordinate of focus, and $x_{r}=\pi \sigma_{w}^{2} / \lambda$ is the Rayleigh diffraction length. $R=x\left\{1+\left[x_{r} /\left(x-x_{f}\right)\right]^{2}\right\}$ is the radius of curvature of the pulse wavefront at $x . \tau$ is the $1 / e$ half pulse width, $t_{\text {peak }}$ is the time delay of envelope peak to the simulation start, and $\phi$ is the carrier envelope phase (CEP). The transverse magnetic field in vacuum can be expressed as $\boldsymbol{B}_{\perp}=\boldsymbol{E}_{\perp} / c$. The longitudinal electric and magnetic fields of the laser pulses are also included in the simulation and their expressions can be derived from Eq. (1) [62].

\section{INJECTION}

2D PIC results of the injection process are shown in Fig. 1. The simulation box has 8000 cells in the $x$ direction and 3000 cells in the $y$ direction, corresponding to a rectangular area of $160.0 \times 120.0 \mu \mathrm{m}^{2}$. The time step is $d t \approx 0.033$ fs. The box starts moving in the $x$ direction with velocity $v_{w} \approx 0.9995 c$ at time $t_{w 0} \approx 500 \mathrm{fs}$. The plasma density has a linear rising ramp starting from $x=50.0 \mu \mathrm{m}$ and ending at $x=51.0 \mu \mathrm{m}$. The electron density is $n_{e}=1.0 \times 10^{18} \mathrm{~cm}^{-3}$, and four macroparticles per cell are loaded where electron density is above zero. Electron temperature is $10 \mathrm{eV}$ and ions are assumed to be fixed background. The laser parameters are $a_{\text {peak } 0}=3.0$, $a_{\text {peak } 1}=4.0, x_{f 0}=60.0 \mu \mathrm{m}, x_{f 1}=115.0 \mu \mathrm{m}, \sigma_{w 0}=24.8 \mu \mathrm{m}$, $\sigma_{w 1}=3.0 \mu \mathrm{m}, t_{\text {peak } 0} \approx 100 \mathrm{fs}, t_{\text {peak } 1} \approx 137 \mathrm{fs}, \tau_{0} \approx 23 \mathrm{fs}$, $\tau_{1} \approx 6 \mathrm{fs}, \phi_{0}=0 \mathrm{rad}$, and $\phi_{1} \approx 4.712 \mathrm{rad}$. Both pulses are polarized in the $y$ direction. Electrons respond to the ponderomotive force of laser pulses, which is expressed as $\boldsymbol{F}_{p} \simeq-\left(m_{e} c / \gamma\right) \nabla\left(\boldsymbol{a}^{2} / 2\right)$. For a laser pulse with Gaussian temporal profiles, the on-axis ponderomotive force is forward during the rising half of the pulse and is backward during the falling half. When the laser pulse hits the plasma boundary, electrons are pushed forward [Fig. 1(a)], and the charge separation field $E_{x}>0$ is generated and becomes larger as the pulse propagates further into the plasma, as depicted in Fig. 1(b). When the electrons slip into the falling half of the pulse, they are decelerated by the ponderomotive force and charge separation field, and eventually they begin moving backward. Some electrons near the VPT then escape into vacuum and a boundary field $E_{x}<0$ is generated [Figs. 1(a) and 1(b)]. As more electrons escape into vacuum, the amplitude of the boundary field becomes larger and some backward moving electrons begin to be decelerated and then accelerated forward, which causes the formation of a thin electron sheet near the VPT, as shown in Figs. 1(a) and 1(e). The injected electrons are located inside the sheet and they experience forward acceleration field, as depicted in Fig. 1(b). With the presence of the boundary field, the electrons near the VPT can stay in the acceleration fields longer than the electrons deeper inside the plasma, and thus they gain larger momentums. However, the initial momentum boost by the boundary field is not enough to make electrons injected in such a tenuous plasma as in our case [Fig. 1(c)]. If the injector was absent, the electrons would slip backward relatively to the wakefields and eventually fall into the deceleration part. Due to the focusing of the injector [Fig. 1(d)], the peak amplitude on axis is rising and the longitudinal length of the bubble is increasing as shown in Figs. 1(a) and 1(e). Thus the initially boosted electrons can stay in the acceleration fields long enough to gain considerable momentum to catch up with the wakefields, as illustrated in Figs. 1(b) and 1(c). The transverse size of the bunch is limited by the modulated wakefields, as shown in Fig. 1(e), and the electrons are moving near the axis with small transverse momentums, so the transverse emittance is very small. The injection area is approximately $2.0 \times 2.0 \mu \mathrm{m}^{2}$, as shown in Fig. 1(d), which is much smaller than that of self-injection [15,23]. 


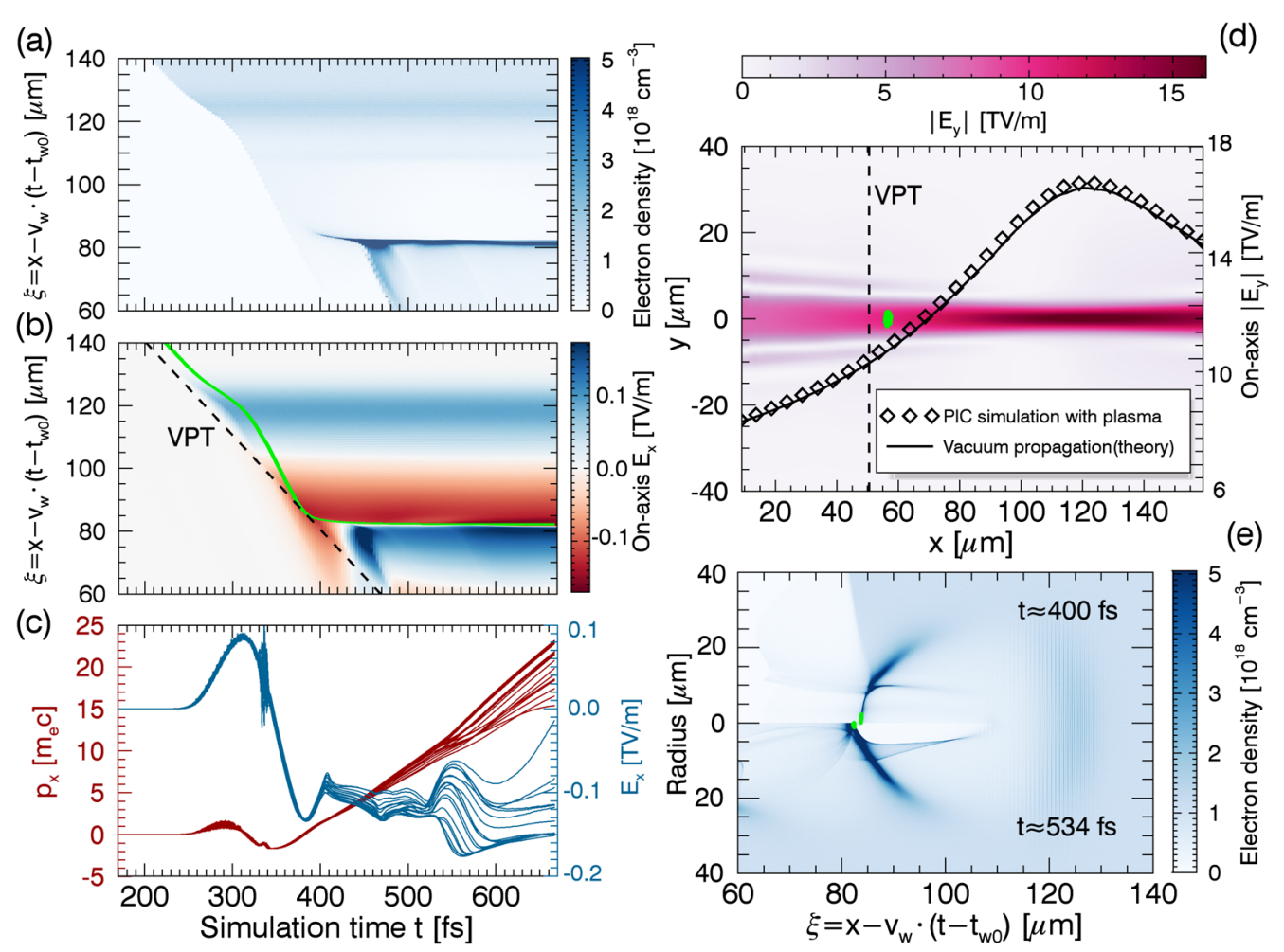

FIG. 1. 2D PIC simulation results. (a) The evolution of on-axis electron density, where $\xi$ is the comoving coordinate. (b) Evolution of on-axis longitudinal electric field $E_{x}$. The green curves are the trajectories of 18 sample macroparticles. (c) Evolution of the longitudinal momentums and experienced longitudinal electric fields of the sample macroparticles from (b). (d) The amplitude of injector pulse along its propagation. The theoretical curve is obtained from Eq. (1). The green dots represent the initial positions of all injected electrons. (e) Electron density distribution in the comoving coordinates $(\xi, r)$ at different simulation times. The green dots are the positions of the sample macroparticles from (b).

The effects of several laser plasma parameters on DPCI are investigated with a series of $2 \mathrm{D}$ simulations. The focusing profile of injector plays a key role in DPCI and electron bunch parameters can be tuned by changing the amplitude, delay and focal spot size of the injector. The peak amplitudes of the injector, $a_{\text {peak } 1}$, were changed from 2.8 to 5.2 while the other parameters were unaltered, the results are depicted in Fig. 2(a). The time delay between the driver and injector, $t_{\text {delay }}=t_{\text {peak } 1}-t_{\text {peak } 0}$, were scanned from 34 to 50 fs keeping the amplitude $a_{\text {peak } 1}=2.8$ and other parameters unchanged, and the results are shown in Fig. 2(b). If the amplitude or delay is too large or too small, the injection is depressed. With $a_{\text {peak } 1}=3.6$ and $t_{\text {delay }} \approx 37 \mathrm{fs}$, the injected charge drops with larger injector focal spot size $\sigma_{w 1}$ as in Fig. 2(c). As the sharp VPT plays a key role in localizing the injection, we also investigated the effects of different lengths of the linear density ramp. With $a_{\text {peak } 1}=3.6, \quad t_{\text {delay }} \approx 37 \mathrm{fs}$ and $\sigma_{w 1}=3 \mu \mathrm{m}$, the ramp length is changed from 1 to $7 \mu \mathrm{m}$ with the ramp center fixed at $x=50.5 \mu \mathrm{m}$. The injection is depressed when longer ramps are used as shown in Fig. 2(d). The charge separation field near the density transition is essential in the injection, and its amplitude is affected by the density difference and ramp length of the transition. Longer ramps can be used for higher plasma densities, but the energies of accelerated electron beams are lower due to shorter dephasing lengths [18]. Sharp density transitions shorter than ten microns are challenging to be realized in experiments but several techniques are proved to be promising such as shocks [37,39], laser machining [63] and cluster jets [64]. The emittance and momentum spread are both positively correlated with the bunch length, as shown in Fig. 2. The tolerance for misalignment is also investigated. It is found that the maximum displacement $\Delta r$ in the first $50 \mu \mathrm{m}$ inside plasma of the injector center from the axis must be smaller than the waist of the injector, i.e., $\Delta r \lesssim \sigma_{w 1}$.

As the 3D laser pulse evolution is different from the 2D case, 3D PIC simulations are performed to verify the injection mechanism. The simulation box has a length of $75 \mu \mathrm{m}$ in the $x$ direction, which is discretized into 1500 cells, and a width of $120 \mu \mathrm{m}$ in both $y$ and $z$ directions, which is discretized into 1200 cells. The time step is $d t \approx 0.083 \mathrm{fs}$. The uniform plasma has a perfect boundary at $x_{0}=10.0 \mu \mathrm{m}$, i.e., electron density is $n_{e}=1.0 \times 10^{18} \cdot H\left(x-x_{0}\right) \mathrm{cm}^{-3}$, where $H(x)$ is the Heaviside step function. There are two microparticles per cell to model the electrons. The simulation box starts moving with velocity $v_{w} \approx 0.9980 c$ at time $t_{w 0} \approx 233 \mathrm{fs}$. The laser parameters are as follows: 

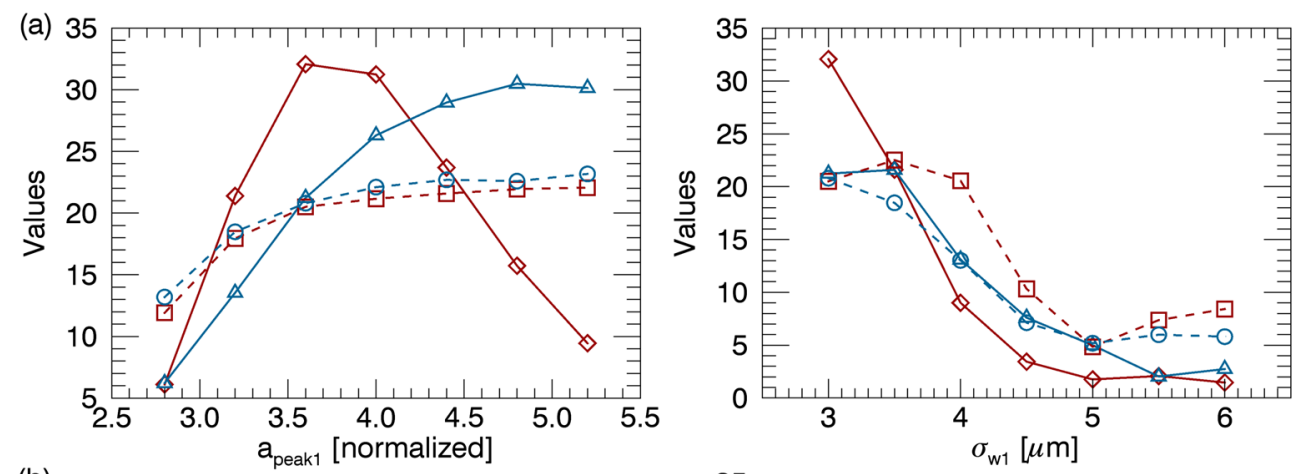

(c)
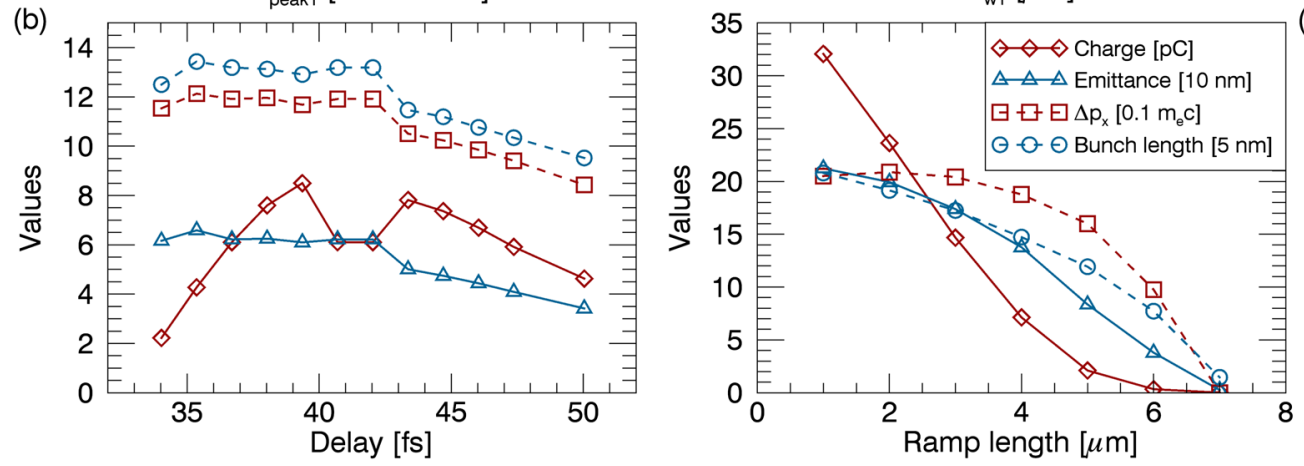

(d)

FIG. 2. Injected charge, rms emittance, rms momentum spread and rms bunch length for different simulation parameters, (a) peak injector amplitude $a_{\text {peak } 1}$, (b) time delay $t_{\text {delay }}$, (c) focal spot size $\sigma_{w 1}$, (d) ramp length. These statistics are obtained by taking all electrons above $7.5 \mathrm{MeV}$ into account at simulation time $t \approx 667 \mathrm{fs}$. The charge is estimated by assuming the width of the simulation box in the $z$ direction is the same as that in $y$.

$a_{\text {peak } 0}=a_{\text {peak } 1}=2.0, \lambda_{0}=\lambda_{1}=0.8 \mu \mathrm{m}, x_{f 0}=20.0 \mu \mathrm{m}$, $x_{f 1}=75.0 \mu \mathrm{m}, \sigma_{w 0}=20.0 \mu \mathrm{m}, \sigma_{w 1}=3.0 \mu \mathrm{m}, t_{\text {peak } 0} \approx 53 \mathrm{fs}$, $t_{\text {peak1 }} \approx 93$ fs. The rest parameters are the same as the $2 \mathrm{D}$ case. Similar to the 2D case, an ultrashort electron bunch is injected in the rear of the bubble [Fig. 3(a)] and it is isolated in phase space, as depicted in Fig. 3(b), indicating that the injection is localized. The phase-space volume of the injected bunch is also well confined, as in Figs. 3(b) and 3(c). The root-mean-square (rms) bunch length is about $0.5 \mu \mathrm{m}$. A negative momentum chirp (electrons in the bunch head have larger momentums than those in the bunch tail) and a relatively small slice energy spread are the

(a)
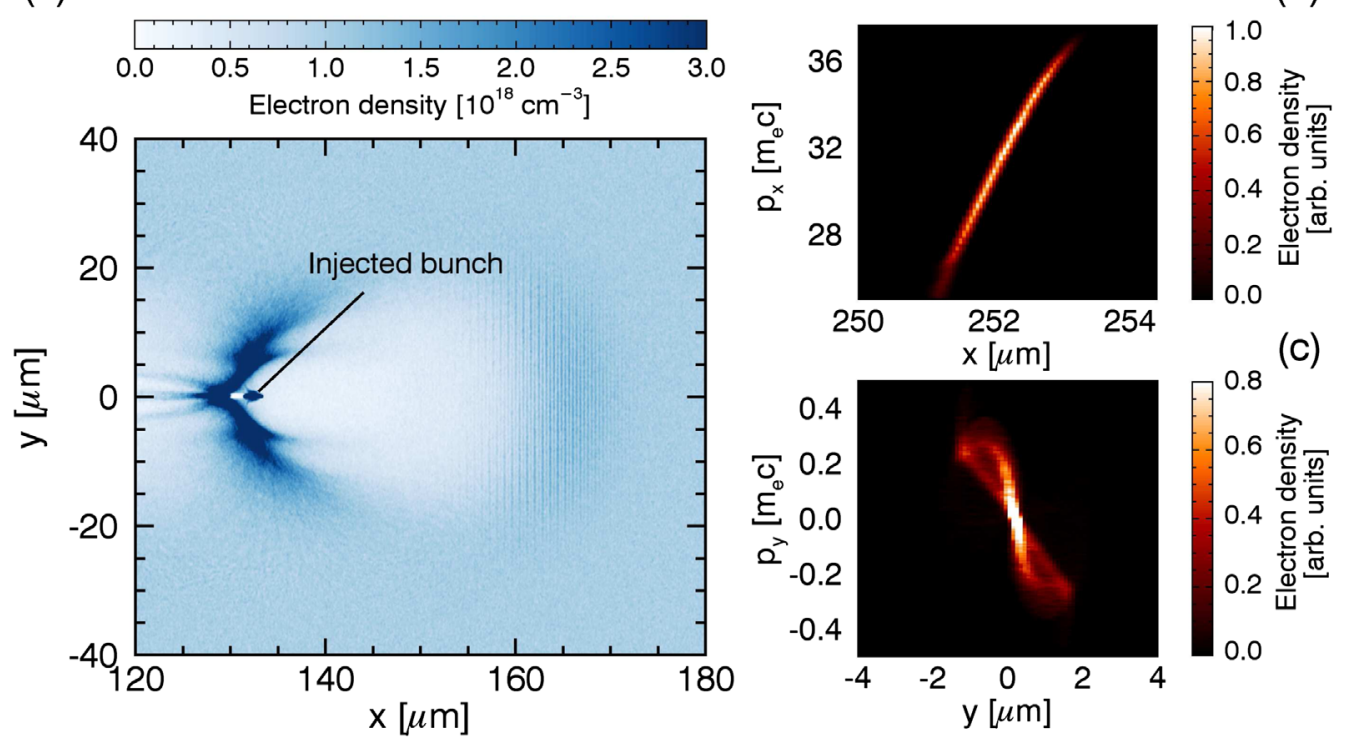

FIG. 3. 3D simulation results for DPCI. (a) Electron density distribution on the $z=0$ plane at $t \approx 600$ fs. (b) Electron longitudinal phase-space distribution at $t \approx 1.00$ ps. (c) Electron transverse phase-space distribution at $t \approx 1.00$ ps. 
features of DPCI, as shown in Fig. 3(b). The phase space along the laser polarization direction is shown in Fig. 3(c), and the normalized rms transverse emittance is $\epsilon_{n}=\sqrt{\left\langle y^{2}\right\rangle\left\langle p_{y}^{2}\right\rangle-\left\langle y p_{y}\right\rangle^{2}} / m_{e} c=89.9 \mathrm{~nm}$, where $\langle\cdot\rangle$ represents averaging over all electrons over $7.5 \mathrm{MeV}$. The phase space of the third dimension is pretty much the same as that of the polarization direction. The total charge over $7.5 \mathrm{MeV}$ is about $2.0 \mathrm{pC}$, the peak current $I$ is about $0.4 \mathrm{kA}$ and the peak brightness is estimated as $B_{n}=$ $2 I / \epsilon_{n}^{2} \approx 1.0 \times 10^{17} \mathrm{~A} \cdot \mathrm{rad}^{-2} \cdot \mathrm{m}^{-2}$, which is as bright as the electron beams driving linac coherent light source (LCLS) [7].

\section{ACCELERATION}

The absolute energy spread of an electron bunch would grow drastically after a long distance inside acceleration field with positive gradient due to the phase-space rotation $[21,41,65]$. In the following discussion, we show that by changing the acceleration gradient with rephasing, the direction of phase-space rotation can be changed and the energy spread can be controlled. For the electron bunch injected with DPCI, the charge is far less than the beam loading limit [66], the longitudinal velocity is close to light velocity, the transverse momentums and slice energy spread can be neglected, so the evolution of energy spread can be derived from the Lorentz equations as

$$
\Delta E \approx \Delta E_{0}-e c k_{E} \cdot \Delta x_{0} \cdot \Delta t,
$$

where $\Delta E$ is the final energy spread, $\Delta E_{0}$ is the initial energy spread, $k_{E}=\partial E_{x} /\left.\partial x\right|_{y=z=0}$ is the slowly varying on-axis slope of the longitudinal electric field, $\Delta x_{0}$ is the bunch length, which is assumed to be constant, and $\Delta t$ is the elapsed time. For the laser plasma wakefields, the longitudinal electric field has a positive gradient inside the low electron density part, and a negative gradient in the high density part [1-4]. The phase-space rotation is clockwise in the positive acceleration gradient and anticlockwise in the negative acceleration gradient. In DPCI mechanism, when the injector is defocusing, the longitudinal length of the plasma bubble shortens and the injected electrons are located in the longitudinal electric field where $k_{E}<0$. The electron bunch is negatively chirped and its energy spread is enlarged due to the negative field gradient. As the electrons are faster than the wakefields, they later advance into the acceleration field with positive gradient and their energy spread is decreased. After energy decompression and compression, the small energy spread can be retained after relatively long distance acceleration and the relative energy spread can be drastically decreased. After its energy spread reaches minimal with a letter " $U$ " shape in longitudinal phase space, the electron bunch becomes positively chirped and the energy spread starts to grow in the positive field gradient $[21,65]$. With typical laser plasma parameters used in LWFA experiments and simulations, the energy spread reaches minimal before the energy reaches $\mathrm{GeV}$, which makes the relative energy spread much larger than the demand of FELs. To further increase the electron energy while maintaining the absolute energy spread, the electron rephasing technique can be adopted to induce multiple energy decompression and compression. 2D simulation results are depicted in Fig. 4 to demonstrate the feasibility of generating $\mathrm{GeV}$ level electron beam with relative energy spread below $0.5 \%$. The simulation box is $80 \mu \mathrm{m}$ in the $x$ direction with 4000 cells and $120 \mu \mathrm{m}$ in the $y$ direction with 3000 cells. The box starts moving with velocity $v_{w} \approx 0.9995 c$ at time $t_{w 0} \approx 284 \mathrm{fs}$. The plasma has a steplike density profile: a linear rising ramp from 50.0 to $51.0 \mu \mathrm{m}$; uniform density of $1.0 \times 10^{18} \mathrm{~cm}^{-3}$ from $51.0 \mu \mathrm{m}$ to $3.0 \mathrm{~mm}$; another rising ramp from 3.0 to $3.5 \mathrm{~mm}$ with density increased to $1.8 \times 10^{18} \mathrm{~cm}^{-3}$; uniform density after $3.5 \mathrm{~mm}$. The laser parameters are the same as before except $a_{\text {peak } 1}=3.6$. The evolution of the on-axis electron density is shown in Fig. 4(a), the rephasing happens at $t \approx 12.0 \mathrm{ps}$. The electrons are moving with velocity close to $c$, the laser pulse is propagating with a smaller group velocity $v_{g}$, and the distance between the laser pulse and electron bunch is shortening. To make the electron bunch experience negative acceleration gradient again, the plasma bubble needs to be shrinked to make the high density part in the rear catch up with the bunch as in Figs. 4(a) and 4(b). As the length of the bubble is positively correlated with the plasma wavelength, thus the rephasing condition can be written as

$$
L \cdot\left(1-\sqrt{\frac{n_{1}}{n_{2}}}\right)=\left(c-v_{g}\right) \cdot \Delta t,
$$

where $L$ is the bubble length before rephasing, $n_{1}$ and $n_{2}$ are the plasma densities before and after rephasing respectively, $\Delta t$ is the acceleration time before rephasing. For a spherical bubble in the matched condition, the bubble length can be estimated as $L=4 \sqrt{a_{\text {peak } 0}} / k_{p}$, where $k_{p}$ is the plasma wave number $[17,18]$. Considering the numeric dispersion due to the discretization, the group velocity is estimated to be $v_{g} \approx 0.9974 c$ [67]. The acceleration length $c \Delta t$ is approximately the length before the second uniform plasma, and the density ratio is estimated to be $n_{2} / n_{1} \approx 1.76$. With the rephasing technique, the dephasing length is lengthened and the average acceleration gradient is enhanced [59]. Electron bunch can experience energy decompression and compression twice depicted in Figs. 4(c) and 4(d), and the final absolute energy spread has not increased much and the relative energy spread has been reduced to about $0.37 \%$ (rms) as shown in Fig. 4(c). Also the charge and emittance will not change too much before and after the rephasing as shown in Fig. 4(c). Some electrons fall behind into the deceleration and defocusing fields when the injector is defocusing and the bubble is 

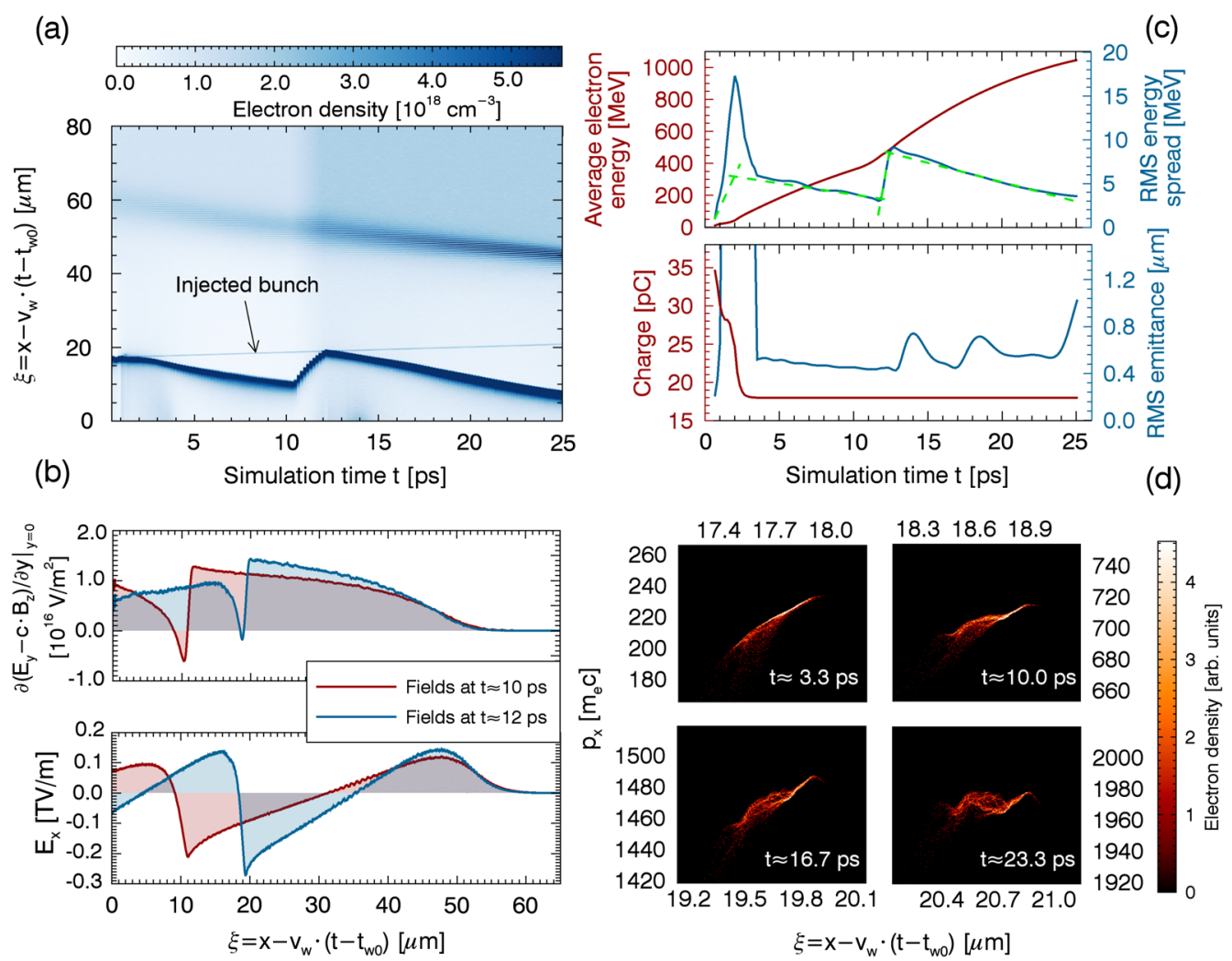

FIG. 4. 2D PIC simulation results of rephasing induced multiple momentum decompression and compression. (a) Evolution of on-axis electron density. (b) The profiles of transverse gradients of focusing fields and longitudinal acceleration fields before and after rephasing. (c) Evolution of electron average energy, rms energy spread, charge and rms emittance. The green dashed lines are the theoretical curves of Eq. (2), where $k_{E}$ is obtained from (b) and $\Delta x_{0}$ is obtained from (d). (d) Electron longitudinal phase space at different times.

shrinking, causing the growth of energy spread and emittance in the early stage of acceleration. If the dephasing was not controlled well, the same would happen to the electron bunch again. In a well-controlled case, no electron is lost and the emittance will not grow too much, as shown in Fig. 4(c). The emittance growth is due to the defocusing fields inside the high electron density part of the wakefields. The on-axis transverse gradient of transverse fields $\partial\left(E_{y}-c \cdot B_{z}\right) /\left.\partial y\right|_{y=0}$ determines the transverse motions of electrons. Electrons experience focusing force when it is above zero and defocusing force when below zero. As depicted in Fig. 4(b), the length and strength of the defocusing part is much smaller than the focusing part. The emittance oscillates due to the sudden change of the focusing force, but later it becomes stable around $0.6 \mu \mathrm{m}$. The emittance growth in the final stage of the simulation is caused by the bunch interacting with laser fields, which can be avoided with some optimizations, and the final emittance can be controlled below $1.0 \mu \mathrm{m}$.

\section{CONCLUSION}

In this paper, we show that with a controlled injection scheme and the phase-space manipulation, the electron energy spread can be reduced to below $0.5 \%$ at $\mathrm{GeV}$ level. In the DPCI scheme, a driver pulse which is below the selfinjection threshold pumps a wakefield for acceleration, and an injector pulse with shorter Rayleigh length is used to trigger injection near a sharp VPT. The injection length can be controlled to be as low as several microns. By changing the parameters of the injector pulse, the parameters of the injected electron bunch can be tuned. The energy compression process is investigated and the compression rate is proportional to the bunch length and the slope of the longitudinal electric field. Using the rephasing technique, the injected bunch can experience multiple energy decompression and compression, thus the absolute energy spread can be maintained while the energy is boosted. Such electron bunches are promising for driving compact FELs and other coherent x-ray sources.

\section{ACKNOWLEDGMENTS}

The authors would like to thank Professor Wei Lu from Tsinghua University and Professor Min Chen from Shanghai Jiao Tong University for useful discussions. The PIC simulatons were carried out in Shanghai Super Computation Center and Max Planck Computing and Data Facility. This work was supported by National Basic 
Research Program of China (Grant No. 2013CBA01502), National Natural Science Foundation of China (Grants No. 11575011 and No. 11535001) and National Grand Instrument Project (2012YQ030142). The EPOCH program was funded by the UK EPSRC Grants No. EP/ G054950/1, No. EP/G056803/1, No. EP/G055165/1 and No. EP/M022463/1.

[1] T. Tajima and J. M. Dawson, Laser Electron Accelerator, Phys. Rev. Lett. 43, 267 (1979).

[2] V. Malka, J. Faure, Y. A. Gauduel, E. Lefebvre, A. Rousse, and K. T. Phuoc, Principles and applications of compact laser-plasma accelerators, Nat. Phys. 4, 447 (2008).

[3] E. Esarey, C. B. Schroeder, and W. P. Leemans, Physics of laser-driven plasma-based electron accelerators, Rev. Mod. Phys. 81, 1229 (2009).

[4] S. M. Hooker, Developments in laser-driven plasma accelerators, Nat. Photonics 7, 775 (2013).

[5] K. Nakajima, Compact X-ray sources: Towards a table-top free-electron laser, Nat. Phys. 4, 92 (2008).

[6] B. W. J. McNeil and N. R. Thompson, X-ray free-electron lasers, Nat. Photonics 4, 814 (2010).

[7] P. Emma et al., First lasing and operation of an ångstromwavelength free-electron laser, Nat. Photonics 4, 641 (2010).

[8] H. T. Kim, K. H. Pae, H. J. Cha, I. J. Kim, T. J. Yu, J. H. Sung, S. K. Lee, T. M. Jeong, and J. Lee, Enhancement of Electron Energy to The Multi-Gev Regime by a Dual-Stage Laser-Wakefield Accelerator Pumped by Petawatt Laser Pulses, Phys. Rev. Lett. 111, 165002 (2013).

[9] X. Wang et al., Quasi-monoenergetic laser-plasma acceleration of electrons to $2 \mathrm{GeV}$, Nat. Commun. 4, 1988 (2013).

[10] W. P. Leemans et al., Multi-GeV Electron Beams from Capillary-Discharge-Guided Subpetawatt Laser Pulses in the Self-Trapping Regime, Phys. Rev. Lett. 113, 245002 (2014).

[11] A. J. Gonsalves et al., Generation and pointing stabilization of multi-GeV electron beams from a laser plasma accelerator driven in a pre-formed plasma waveguide, Phys. Plasmas 22, 056703 (2015).

[12] I. Kostyukov, A. Pukhov, and S. Kiselev, Phenomenological theory of laser-plasma interaction in "bubble" regime, Phys. Plasmas 11, 5256 (2004).

[13] I. Kostyukov, E. Nerush, A. Pukhov, and V. Seredov, Electron Self-Injection in Multidimensional RelativisticPlasma Wake Fields, Phys. Rev. Lett. 103, 175003 (2009).

[14] A. G. R. Thomas, Scalings for radiation from plasma bubbles, Phys. Plasmas 17, 056708 (2010).

[15] M. R. Islam et al., Near-threshold electron injection in the laser-plasma wakefield accelerator leading to femtosecond bunches, New J. Phys. 17, 093033 (2015).

[16] W. Lu, C. Huang, M. Zhou, W. B. Mori, and T. Katsouleas, Nonlinear Theory for Relativistic Plasma Wakefields in the Blowout Regime, Phys. Rev. Lett. 96, 165002 (2006).

[17] W. Lu, C. Huang, M. Zhou, M. Tzoufras, F. S. Tsung, W. B. Mori, and T. Katsouleas, A nonlinear theory for multidimensional relativistic plasma wave wakefields, Phys. Plasmas 13, 056709 (2006).

[18] W. Lu, M. Tzoufras, C. Joshi, F. S. Tsung, W. B. Mori, J. Vieira, R. A. Fonseca, and L. O. Silva, Generating multi$\mathrm{GeV}$ electron bunches using single stage laser wakefield acceleration in a 3D nonlinear regime, Phys. Rev. ST Accel. Beams 10, 061301 (2007).

[19] S. Kalmykov, S. A. Yi, V. Khudik, and G. Shvets, Electron Self-Injection and Trapping into an Evolving Plasma Bubble, Phys. Rev. Lett. 103, 135004 (2009).

[20] S. Y. Kalmykov, A. Beck, S. A. Yi, V. N. Khudik, M. C. Downer, E. Lefebvre, B. A. Shadwick, and D.P. Umstadter, Electron self-injection into an evolving plasma bubble: Quasi-monoenergetic laser-plasma acceleration in the blowout regime, Phys. Plasmas 18, 056704 (2011).

[21] S. Y. Kalmykov, S. A. Yi, A. Beck, A. F. Lifschitz, X. Davoine, E. Lefebvre, V. Khudik, G. Shvets, and M. C. Downer, Dark-current-free petawatt laser-driven wakefield accelerator based on electron self-injection into an expanding plasma bubble, Plasma Phys. Controlled Fusion 53, 014006 (2011).

[22] C. Benedetti, C. B. Schroeder, E. Esarey, F. Rossi, and W. P. Leemans, Numerical investigation of electron selfinjection in the nonlinear bubble regime, Phys. Plasmas 20, 103108 (2013).

[23] B. M. Cowan, S. Y. Kalmykov, A. Beck, X. Davoine, K. Bunkers, A. F. Lifschitz, E. Lefebvre, D. L. Bruhwiler, B. A. Shadwick, and D. P. Umstadter, Computationally efficient methods for modelling laser wakefield acceleration in the blowout regime, J. Plasma Phys. 78, 469 (2012).

[24] S. P. D. Mangles et al., Monoenergetic beams of relativistic electrons from intense laser-plasma interactions, Nature (London) 431, 535 (2004).

[25] C. G. R. Geddes, Cs. Toth, J. van Tilborg, E. Esarey, C. B. Schroeder, D. Bruhwiler, C. Nieter, J. Cary, and W. P. Leemans, High-quality electron beams from a laser wakefield accelerator using plasma-channel guiding, Nature (London) 431, 538 (2004).

[26] J. Faure, Y. Glinec, A. Pukhov, S. Kiselev, S. Gordienko, E. Lefebvre, J.-P. Rousseau, F. Burgy, and V. Malka, A laserplasma accelerator producing monoenergetic electron beams, Nature (London) 431, 541 (2004).

[27] W. P. Leemans, B. Nagler, A. J. Gonsalves, Cs. Tóth, K. Nakamura, C. G. R. Geddes, E. Esarey, C. B. Schroeder, and S. M. Hooker, GeV electron beams from a centimetrescale accelerator, Nat. Phys. 2, 696 (2006).

[28] N. A. M. Hafz et al., Stable generation of GeV-class electron beams from self-guided laser-plasma channels, Nat. Photonics 2, 571 (2008).

[29] D. H. Froula et al., Measurements of the Critical Power for Self-Injection of Electrons in a Laser Wakefield Accelerator, Phys. Rev. Lett. 103, 215006 (2009).

[30] K. Schmid et al., Few-Cycle Laser-Driven Electron Acceleration, Phys. Rev. Lett. 102, 124801 (2009).

[31] S. P. D. Mangles, G. Genoud, M. S. Bloom, M. Burza, Z. Najmudin, A. Persson, K. Svensson, A. G. R. Thomas, and C.-G. Wahlström, Self-injection threshold in self-guided laser wakefield accelerators, Phys. Rev. ST Accel. Beams 15, 011302 (2012). 
[32] S. Banerjee et al., Stable, tunable, quasimonoenergetic electron beams produced in a laser wakefield near the threshold for self-injection, Phys. Rev. ST Accel. Beams 16, 031302 (2013).

[33] S. Fritzler, E. Lefebvre, V. Malka, F. Burgy, A. E. Dangor, K. Krushelnick, S. P. D. Mangles, Z. Najmudin, J.-P. Rousseau, and B. Walton, Emittance Measurements of a Laser-Wakefield-Accelerated Electron Beam, Phys. Rev. Lett. 92, 165006 (2004).

[34] S. Kneip et al., Characterization of transverse beam emittance of electrons from a laser-plasma wakefield accelerator in the bubble regime using betatron $\mathrm{x}$-ray radiation, Phys. Rev. ST Accel. Beams 15, 021302 (2012).

[35] G. R. Plateau et al., Low-Emittance Electron Bunches from a Laser-Plasma Accelerator Measured using Single-Shot X-Ray Spectroscopy, Phys. Rev. Lett. 109, 064802 (2012).

[36] C. G. R. Geddes, K. Nakamura, G. R. Plateau, Cs. Toth, E. Cormier-Michel, E. Esarey, C. B. Schroeder, J. R. Cary, and W. P. Leemans, Plasma-Density-Gradient Injection of Low Absolute-Momentum-Spread Electron Bunches, Phys. Rev. Lett. 100, 215004 (2008).

[37] K. Schmid, A. Buck, C. M. S. Sears, J. M. Mikhailova, R. Tautz, D. Herrmann, M. Geissler, F. Krausz, and L. Veisz, Density-transition based electron injector for laser driven wakefield accelerators, Phys. Rev. ST Accel. Beams 13, 091301 (2010).

[38] F. Y. Li, Z. M. Sheng, Y. Liu, J. Meyer-ter-Vehn, W. B. Mori, W. Lu, and J. Zhang, Dense Attosecond Electron Sheets from Laser Wakefields Using an Up-Ramp Density Transition, Phys. Rev. Lett. 110, 135002 (2013).

[39] A. Buck et al., Shock-Front Injector for High-Quality Laser-Plasma Acceleration, Phys. Rev. Lett. 110, 185006 (2013).

[40] J. Faure, C. Rechatin, A. Norlin, A. Lifschitz, Y. Glinec, and V. Malka, Controlled injection and acceleration of electrons in plasma wakefields by colliding laser pulses, Nature (London) 444, 737 (2006).

[41] X. Davoine, E. Lefebvre, C. Rechatin, J. Faure, and V. Malka, Cold Optical Injection Producing Monoenergetic, Multi-GeV Electron Bunches, Phys. Rev. Lett. 102, 065001 (2009).

[42] H. Kotaki et al., Electron Optical Injection with Head-On and Countercrossing Colliding Laser Pulses, Phys. Rev. Lett. 103, 194803 (2009).

[43] R. Lehe, A. F. Lifschitz, X. Davoine, C. Thaury, and V. Malka, Optical Transverse Injection in Laser-Plasma Acceleration, Phys. Rev. Lett. 111, 085005 (2013).

[44] M. Chen et al., Electron injection and emittance control by transverse colliding pulses in a laser-plasma accelerator, Phys. Rev. ST Accel. Beams 17, 051303 (2014).

[45] A. Pak, K. A. Marsh, S. F. Martins, W. Lu, W. B. Mori, and C. Joshi, Injection and Trapping of Tunnel-Ionized Electrons into Laser-Produced Wakes, Phys. Rev. Lett. 104, 025003 (2010).

[46] C. E. Clayton et al., Self-Guided Laser Wakefield Acceleration beyond $1 \mathrm{GeV}$ Using Ionization-Induced Injection, Phys. Rev. Lett. 105, 105003 (2010).

[47] H. Lu et al., Laser wakefield acceleration of electron beams beyond $1 \mathrm{GeV}$ from an ablative capillary discharge waveguide, Appl. Phys. Lett. 99, 091502 (2011).
[48] F. Li et al., Generating High-Brightness Electron Beams via Ionization Injection by Transverse Colliding Lasers in a Plasma-Wakefield Accelerator, Phys. Rev. Lett. 111, 015003 (2013).

[49] N. Bourgeois, J. Cowley, and S. M. Hooker, Two-Pulse Ionization Injection into Quasilinear LaserWakefields, Phys. Rev. Lett. 111, 155004 (2013).

[50] L.-L. Yu, E. Esarey, C. B. Schroeder, J.-L. Vay, C. Benedetti, C. G. R. Geddes, M. Chen, and W. P. Leemans, Two-Color Laser-Ionization Injection, Phys. Rev. Lett. 112, 125001 (2014).

[51] X. L. Xu et al., Phase-Space Dynamics of Ionization Injection in Plasma-Based Accelerators, Phys. Rev. Lett. 112, 035003 (2014).

[52] X. L. Xu et al., Low emittance electron beam generation from a laser wakefield accelerator using two laser pulses with different wavelengths, Phys. Rev. ST Accel. Beams 17, 061301 (2014).

[53] M. Zeng, M. Chen, L. L. Yu, W. B. Mori, Z. M. Sheng, B. Hidding, D. A. Jaroszynski, and J. Zhang, Multichromatic Narrow-Energy-Spread Electron Bunches from LaserWakefield Acceleration with Dual-Color Lasers, Phys. Rev. Lett. 114, 084801 (2015).

[54] M. Mirzaie et al., Demonstration of self-truncated ionization injection for GeV electron beams, Sci. Rep. 5, 14659 (2015).

[55] A. G. R. Thomas et al., Monoenergetic Electronic Beam Production Using Dual Collinear Laser Pulses, Phys. Rev. Lett. 100, 255002 (2008).

[56] P. Sprangle, B. Hafizi, J. R. Peñano, R. F. Hubbard, A. Ting, C. I. Moore, D. F. Gordon, A. Zigler, D. Kaganovich, and T.M. Antonsen, Wakefield generation and $\mathrm{GeV}$ acceleration in tapered plasma channels, Phys. Rev. E 63, 056405 (2001).

[57] W. Rittershofer, C. B. Schroeder, E. Esarey, F. J. Grüner, and W. P. Leemans, Tapered plasma channels to phase-lock accelerating and focusing forces in laser-plasma accelerators, Phys. Plasmas 17, 063104 (2010).

[58] M. S. Hur and H. Suk, Numerical study of $1.1 \mathrm{GeV}$ electron acceleration over a-few-millimeter-long plasma with a tapered density, Phys. Plasmas 18, 033102 (2011).

[59] E. Guillaume et al., Electron Rephasing in a Laser-Wakefield Accelerator, Phys. Rev. Lett. 115, 155002 (2015).

[60] M. Chen et al., Development and application of multidimensional particle-in-cellcodes, Chin. J. Comput. Phys. 25, 43 (2008).

[61] T. D. Arber et al., Contemporary particle-in-cell approach to laser-plasma modelling, Plasma Phys. Controlled Fusion 57, 113001 (2015).

[62] S. Masuda, M. Kando, H. Kotaki, and K. Nakajima, Suppression of electron scattering by the longitudinal components of tightly focused laser fields, Phys. Plasmas 12, 013102 (2005).

[63] C.-T. Hsieh, C.-M. Huang, C.-L. Chang, Y.-C. Ho, Y.-S. Chen, J.-Y. Lin, J. Wang, and S.-Y. Chen, Tomography of Injection and Acceleration of Monoenergetic Electrons in a Laser-Wakefield Accelerator, Phys. Rev. Lett. 96, 095001 (2006). 
[64] H. Sheng, K. Y. Kim, V. Kumarappan, B. D. Layer, and H. M. Milchberg, Plasma waveguides efficiently generated by Bessel beams in elongated cluster gas jets, Phys. Rev. E 72, 036411 (2005).

[65] F. S. Tsung, R. Narang, W. B. Mori, C. Joshi, R. A. Fonseca, and L. O. Silva, Near-GeV-Energy Laser-Wakefield Acceleration of Self-Injected Electrons in a Centimeter-Scale Plasma Channel, Phys. Rev. Lett. 93, 185002 (2004).
[66] C. Rechatin, X. Davoine, A. Lifschitz, A. Ben Ismail, J. Lim, E. Lefebvre, J. Faure, and V. Malka, Observation of Beam Loading in a Laser-Plasma Accelerator, Phys. Rev. Lett. 103, 194804 (2009).

[67] B. M. Cowan, D. L. Bruhwiler, J. R. Cary, E. CormierMichel, and C. G. R. Geddes, Generalized algorithm for control of numerical dispersion in explicit time-domain electromagnetic simulations, Phys. Rev. ST Accel. Beams 16, 041303 (2013). 